\title{
Clima social familiar y adaptación de conducta en estudiantes del quinto grado de secundaria de la Gran Unidad Escolar Las Mercedes, Juliaca - 2015
Family social climate and behavioral adaptation in fifth grade students of the Great Las Mercedes School Unit, Juliaca - 2015

\author{
Aquize Anco, Eddy Wildmar; Nuñez Paja, José Vilmor \\ EP de Psicología, Facultad de Ciencias de la Salud, Universidad Peruana Unión \\ Recibido el 10 de mayo del 2016 - Aceptado el 26 de junio del 2016
}

\begin{abstract}
Resumen
El objetivo de esta investigación fue determinar el grado de relación entre el clima social familiar y la adaptación de conducta en estudiantes del quinto grado de secundaria de la Gran Unidad Escolar Las Mercedes de la ciudad de Juliaca, inscritos en el año 2015. La población estuvo conformada por 307 estudiantes entre varones y mujeres de edades que oscilan de 14 a 16 años, sin embargo, se dispuso a trabajar con una muestra probabilística estratificada. La metodología de la presente investigación corresponde a un diseño no experimental de corte transversal y de tipo descriptivo correlacional. Los instrumentos utilizados fueron la escala de clima social familiar (en sus siglas FESS) creado por Moss, que cuenta con 90 ítems de elección dicotómica y el inventario de adaptación de conducta (IAC) con 123 ítems. Los resultados indican que el 49.1\% de los estudiantes encuestados presentan un nivel promedio con relación al clima social familiar y el 14.5\% un nivel malo; asimismo, el $53.6 \%$ muestra dificultades en su adaptación de conducta y tan solo el 9\% muestra una satisfactoria adaptación. Otro resultado principal nos muestra el nivel de correlación entre las variables de estudio: clima social familiar y adaptación de conducta $(r=, 514 \mathrm{p}<0.05)$, clima social y adaptación personal $(\mathrm{r}=, 347 \mathrm{p}<0,05)$, clima social familiar y adaptación familiar $(\mathrm{r}=520 \mathrm{p}<0.05)$, clima social familiar y adaptación social $(\mathrm{r}=, 288 \mathrm{p}<0.05)$. Sin embargo, se encontró una nula correlación entre el clima social familiar y la adaptación educativa $(\mathrm{r}=-, 005 \mathrm{p}$ $>0,05)$, lo cual indica que el niño adquiere patrones de socialización en la familia que terminan de desarrollarse en otros grupos pares durante la adolescencia.
\end{abstract}

Palabras clave: Clima social familiar, adaptación de conducta y adolescencia.

\begin{abstract}
The aim of this research was to determine the degree of relationship between the family social climate and behavioral adaptation in fifth grade students of the Great Las Mercedes School Unit of the city of Juliaca, registered in the year 2015. The population was conformed By 307 students among males and females ranging in age from 14 to 16 years, however, was prepared to work with a stratified probabilistic sample. The methodology of the present research corresponds to a non - experimental cross - sectional design and descriptive correlational type. The instruments used were the family social climate scale (in its initials FESS) created by Moss that has 90 items of dichotomous choice and the inventory of behavior adaptation (IAC) with 123 items. The results indicate that $49.1 \%$ of the students surveyed presented an average level in relation to the family social climate and $14.5 \%$ had a bad level. Likewise, 53.6\% showed difficulties in their behavioral adaptation and only 9\% showed a satisfactory level adaptation. Another main result shows the correlation between the study variables: family social climate and behavioral adaptation $(\mathrm{r}=, 514 \mathrm{p}<0.05)$, social climate and personal adaptation $(\mathrm{r}=, 347 \mathrm{p}<0.05)$, climate $(\mathrm{R}=520 \mathrm{p}<0.05)$, family social climate and social adaptation $(\mathrm{r}=, 288 \mathrm{p}<0.05)$. However, there was no correlation between family social climate and educational adaptation $(r=-0.005 \mathrm{P}>0.05)$, which indicates that the child acquires patterns of socialization in the family that end up developing in other peer groups in adolescence.
\end{abstract}

Keywords: Family social climate, Adaptation of behavior and Adolescence.

Correspondencia al autor:

email: eddy.wildmar@gmail.com 


\section{Introducción}

La Organización Mundial de la Salud (OMS, 2016) refiere que cerca de 350 millones de personas en el mundo presentan cuadros de depresión, y cada año se presentan 800000 suicidios por esta causa.

Sausa (2014) refiere que, en el año 2013, el Perú registró 334 suicidios teniendo un $17 \%$ de casos de suicidio en menores de 18 años, los cuales son generados por violencia familiar, quiebra de contactos de familia, ruptura de matrimonios, abandono de los padres o familias ausentes y ciberbullying.

En cuanto a la familia se refiere, el Instituto Nacional de Estadística e Informática [INEI] (2015), describe que el $39,9 \%$ de los adolescentes empadronados en la zona sur del Callao en el año 2014, indican que los padres los corrigen llamándoles la atención; el 29,0\% indica que sus padres conversan para corregirlos, y el $26,7 \%$ de los adolescentes tienen al menos un padre que utiliza golpes, cachetadas, puños y patadas. Asimismo, un $24,7 \%$ indica haber recibido insultos o lisuras por parte de sus padres.

Una escasa cohesión familiar en el clima social familiar y la ausencia de pertenecer a un grupo social en el adolescente son fenómenos asociados a la ideación suicida o depresión (Toro, 2010). Por otro lado, investigaciones como las que realizó Ethel y Godiel (2012), describen que el bajo nivel del clima social familiar posee una fuerte conexión con la población de adolescentes embarazadas en la ciudad de Tacna. Teniendo en cuenta lo anterior, en el año 2013, el departamento de Puno poseía un porcentaje de $7.8 \%$ de la población adolescente que estaba embarazada. Esta cifra mutó a un $12.3 \%$ tan solo en el año 2014 (INEI, 2015). Es claro que la familia es un factor importante que permite la prevención o genera problemas sociales, los cuales se proyectan en la vida del adolescente peruano.

Se espera que la familia sea el grupo primario de apoyo, que protege, inculca valores, y fomenta el desarrollo de sus miembros, sin embargo, muchos de estos poseen un clima familiar desfavorable o inadecuado, que puede agravar el desarrollo de hijos adolescentes. Para Orantes (2011), una mala adaptación conduce al adolescente a dos problemas frecuentes, uno de ellos es la depresión, puesto que el adolescente no logra adaptarse a una situación nueva o abrumadora. Asimismo, refiere que otro problema común es la indefensión aprendida, la cual constituye una falta de motivación, que concluye en una resignación de la vida donde no hay nada que hacer, ni ahora ni nunca, dejando ambiciones de lado, la emancipación y una necesaria autorrealización del individuo.

Otra óptica sobre el estado de los adolescentes en el Perú lo informa Daly (2015) en el periódico El Comercio, quien expone que existe una población de 1869 internos menores de edad recluidos en institutos de reinserción juvenil, de los cuales 889 adolescentes son recluidos por motivos de robo agravado y robo en los departamentos de Piura, Lambayeque, La Libertad, Trujillo, Lima, Ucayali, Junín, Cusco y Arequipa. Estas estadísticas dan a conocer solamente una pequeña fracción sobre los problemas que los adolescentes en nuestro país atraviesan.

El diccionario de la Real Academia Española nos proporciona un significado sobre la adolescencia: "Periodo de la vida humana que sigue a la niñez y precede a la juventud" (Real Academia Española [DRAE], 2015). Sin embargo, la adolescencia va más allá de una etapa de transición de niñez a adultez caracterizada por cambios físicos, psicológicos y sociales, llena de situaciones críticas: la búsqueda de identidad, la competencia social, el narcisismo adolescente, la separación o emancipación, son solamente algunas de ellas (Ochoa, 2008).

Investigaciones como Ethel y Godiel (2012), Toro (2010), Orantes (2011), Daly (2015) y Sausa (2014), atribuyen los problemas sociales anteriormente mencionados a un inadecuado clima social familiar y escasos recursos de adaptación en el adolescente. En los últimos años se dio mucha más relevancia a problemas sociales como depresión, embarazos no deseados, fracasos escolares, conductas antisociales entre otras, pues hay escasas investigaciones que dirigen a aquellos factores que los provocan. Es por ello que se realiza la presente investigación, la cual no se limita a estudiar el factor asociado, sino más bien si este puede variar por el comportamiento de otro.

De la misma manera Moos y Moss (1981) indican que el clima social familiar consta de tres dimensiones que contienen áreas relacionadas íntimamente. Una de ellas es la dimensión de relación, en la que se observa la forma cómo los miembros de la familia se relacionan entre sí, con relación a su expresividad y su cohesión, así como también el nivel de conflicto y la resolución de conflictos de cada miembro que conforma la familia.

Roth (2000) refiere que el clima social familiar posee el sustento teórico de la psicología ambiental, que no 
considera al hombre como un producto pasivo de su ambiente sino como un ser orientado hacia metas que actúan sobre su medio ambiente y, al hacerlo, también recibe influencia, a esto se denomina intercambio dinámico.

Distintos autores han coincidido que las personas interactúan en un ambiente, el cual influye en sus cogniciones, conductas, emociones, pues es el mismo producto quien actúa como agente de cambio (Proshansky citado por Roth, 2000).

Para Moss (citado por Santos, 2012), refiere que el clima social familiar es una atmósfera donde se describe las características e instituciones de un determinado grupo situado sobre un ambiente, y que además son tres las dimensiones o atributos afectivos que hay que tener en cuenta. Estas dimensiones están plasmadas en la Escala del Clima Social en la Familia. a) Dimensión relación que valora el grado de comunicación, en su libre expresión y su interacción conflictiva que la caracteriza. Esta dimensión está integrada por 3 subescalas, que son la cohesión, expresividad y conflicto. b) Dimensión desarrollo que valora la importancia que tienen dentro de la familia ciertos procesos de desarrollo que pueden ser fomentados, o no, por la vida en común. Esta dimensión comprende las subescalas de autonomía, actuación, intelectual, cultural, social, recreativa y moralidad religiosa. c) Dimensión estabilidad que proporciona información sobre la estructura y organización de la familia y sobre el grado de control que normalmente ejercen unos miembros de la familia sobre otros, la conforman 2 subescalas: organización y control.

Vera, Morales, y Vera (2005), indican que un clima sin orientación se asocia a una autopercepción negativa del niño y de restricciones por la maternidad y presentan las medias más bajas de desarrollo cognitivo. Asimismo, el clima cohesivo se asocia con mucha frecuencia, con menos estrés en la madre por la crianza y los puntajes más altos de desarrollo cognitivo. García (2005) refiere que todo organismo tiene que adaptarse al medio en el cual se desenvuelve, puesto que la adaptación es un proceso de supervivencia que constituyen la asimilación y la organización, pues se presume que la asimilación es la integración de nuevos elementos al organismo, para que después este los organice a esquemas previamente establecidos, pues el organismo actúa en un ambiente cambiante y este al adaptarse cambia a la vez su ambiente.
Para Gutiérrez (2005) la adaptación posee un concepto que Piaget propone, resultando en la construcción de nuevas estructuras biológicas y psicológicas que sean coherentes desde una óptica interna, ajustadas al medio externo, y que serán modificadas mediante procesos necesarios e inesperados, que se producen mediante la interacción del individuo con su ambiente, adquiriendo mecanismos beneficiosos para su supervivencia los cuales se organizarán al sistema del individuo en general.

Sin embargo, la adaptación de conducta posee un concepto complementario a lo que se indica, el cual consiste en la capacidad o incapacidad del ser humano para modificar su conducta acorde con las exigencias del ambiente que está en constante cambio. Asimismo, la exitosa adaptación comprende factores tales como las habilidades, actitudes y condiciones físicas que la persona posee, para actuar conforme con lo que desea hacer, respecto a lo que el medio exige, adquiriendo la nomenclatura de equilibrio. De igual forma las situaciones que exigen adaptación al individuo cambian acorde con el mundo contemporáneo en el cual vivimos, puesto que ahora, la calidad de vida y la felicidad son más importantes que suplir las necesidades biológicas como lo era en la Edad Antigua (Sarason \& Sarason, 2006).

Para Díaz (2013) la etapa en la que se hace más resaltante la adaptación de conducta es en la adolescencia, puesto que es posible apreciar la importancia del ambiente social en el que se desarrolla el adolescente, el cual influye grandemente en la formación de la identidad propia, intentando lograr un acuerdo armónico entre ellos mismos y el medio ambiente, desarrollando su concepto del yo, donde la asimilación e integración serán determinantes en la conducta social y la personalidad del adolescente, la que proyectará durante su edad adulta.

\section{Método}

Previa aprobación pertinente de la presente investigación, y en coordinación con los directivos de la Universidad Peruana Unión Filial Juliaca, se presentó una solicitud para obtener la autorización a fin de aplicar la investigación a los estudiantes del nivel secundario de la Gran Unidad Escolar Las Mercedes. Asimismo, acorde con el código de ética del profesional psicólogo, artículos 79 y 81 del título Actividades de Investigación, donde, en calidad de investigadores, demanda cuidar y salvaguardar la ética del investigador, la institución deberá ser informada de todas las activida- 
des a desarrollar con relación a dicha investigación. Seguidamente se pedirán voluntarios, esto acorde con el artículo 83 y 85 , donde el investigador deberá informar al participante voluntario sobre el objetivo de la investigación, establecer acuerdos y compromisos que debemos respetar. A su vez, de acuerdo con el artículo 87 del mismo título, se deberá informar a los participantes el destino de los resultados. Los resultados fueron procesados por el paquete estadístico SPSS 20 para obtener la confiabilidad de Cronbach y la correlación de Pearson.

\section{Participantes}

Los participantes poseían una edad que oscilaba entre los 14 a 15 años, entre varones y mujeres inscritos en la Gran Unidad Escolar Las Mercedes de la ciudad de Juliaca, una ciudad caracterizada por poseer muchas familias dedicadas al comercio.

\section{Instrumentos}

Los instrumentos utilizados fueron el cuestionario de clima social familiar (FES) creado por R. H. Moos y Trickett y estandarizado para Lima Metropolitana en el año 1993 por César Ruiz Alva. Este test consta de 90 ítems de elección dicotómica, que mide las relaciones intrafamiliares. Asimismo se consideró aplicar el inventario de adaptación de conducta que consta de 123 ítems para medir la capacidad de adaptar la conducta a situaciones nuevas en el adolescente, por Victoria de la Cruz y Agustín Cordero, en el año de 1990, y estandarizado por César Ruiz Alva en el año de 1995.

\section{Análisis de datos}

Los resultados fueron procesados por el paquete estadístico SPSS 20 para obtener la confiabilidad de Cronbach, y la correlación de Pearson para variables cuantitativas.

\section{Resultados y Discusión}

Con relación al nivel de clima social familiar, el $49,1 \%$ presenta un nivel promedio, el $14,5 \%$ presenta un nivel malo, el 13,6\% presenta un nivel tendiente a bueno. Seguidamente, un 10\% indica un buen clima social familiar, el $6.4 \%$ muestra un nivel excelente $y$, finalmente, otro $6,4 \%$ representa a estudiantes con un clima social familiar deficitario. Por otro lado, se estima que el 53.6\% de los estudiantes indican un nivel bajo, y con ello indican dificultades en su adaptación; asimismo, el $45,5 \%$ revela un nivel medio, lo que se entiende como una normal adaptación en su conducta; y tan solo el 0,9\% manifiesta una satisfactoria adaptación de conducta con un nivel alto.

La tabla 1 indica las correlaciones entre el clima social familiar y las dimensiones de adaptación de conducta; clima social familiar y adaptación de conducta en su dimensión personal $(\mathrm{r}=, 347 ; \mathrm{p}<0,05)$, clima social familiar y adaptación de conducta en su dimensión adaptación familiar $(r=520 ; \mathrm{p}<0,05)$, clima social familiar y adaptación de conducta en su dimensión adaptación social $(r=, 288 ; p<0,05)$, coeficientes que indican una relación directa y significativa. Por otro lado, un coeficiente de correlación $(r=,-0,05 ; \mathrm{p}>$ $0,05)$ indica una nula correlación entre las variables clima social familiar y adaptación de conducta en su dimensión adaptación educativa.

Tabla 1

Coeficientes de correlación entre la variable clima social familiar y las dimensiones de la variable adaptación y conducta.

\begin{tabular}{llccccc}
\hline & $\begin{array}{c}\text { Dimensión } \\
\text { Personal }\end{array}$ & $\begin{array}{c}\text { Dimensión } \\
\text { Familiar }\end{array}$ & $\begin{array}{c}\text { Dimensión } \\
\text { Educativa }\end{array}$ & $\begin{array}{c}\text { Dimensión } \\
\text { Social }\end{array}$ & $\begin{array}{c}\text { Adaptación de } \\
\text { Conducta }\end{array}$ \\
\hline $\begin{array}{l}\text { Clima social } \\
\text { familiar }\end{array}$ & $\begin{array}{l}\text { Correlación de } \\
\text { Pearson }\end{array}$ &, $347^{* *}$ &, $520^{* *}$ &,- 005 &, $288^{* *}$ &, $514^{* *}$ \\
& Sig. (bilateral) &, 000 &, 000 &, 956 &, 002 &, 000 \\
& $\mathrm{~N}$ & 110 & 110 & 110 & 110 & 110 \\
\hline
\end{tabular}

**. La correlación es significativa en el nivel 0,01 ( 2 colas).

*. La correlación es significativa en el nivel 0,05 (2 colas).

Muchas teorías han constituido la importancia de la familia en el desarrollo del niño, pero al desarrollarse, este grupo principalmente llamado primario, constituye un pilar de los muchos que el adolescente ahora tiene, entre ellos la escuela y los amigos, donde la amistad se convierte ahora en una prioridad por ser el grupo en el que el adolescente culminará su desarrollo social; pasa lo mismo al desear entender el desarrollo del adolescente en su entorno socioeducativo (Toro, 2010). Asimismo, la filosofía cristiana indica que los jóvenes no son seres aislados, sino más bien complementarios unos a los otros, por ello apuesta por el internado escolar, donde el 
adolescente tiene más probabilidades de desarrollo que los harán útiles y felices para la vida posterior (White, 1967).

En el presente estudio se halló que existe correlación directa y significativa entre el clima social familiar y la adaptación de conducta, es decir, a mayor nivel de clima social familiar mayor adaptación de conducta. Este resultado concuerda con los hallazgos de Verdugo y otros (2014), Pichardo, Fernández, y Amezcua (2002) y Vargas (2009), quienes encontraron también una correlación directa y significativa entre el clima social y la adaptación de conducta.

Los resultados de la presente investigación muestran que los estudiantes presentan porcentajes elevados de clima social familiar en los niveles de promedio bueno y malo, pues Juliaca es considerada el eje de la macro región sur, donde la mayoría de los juliaqueños son una población económicamente activa, quienes adoptan como prioridad el comercio, en lugar de aquellas actividades que fomenten un adecuado clima social familiar (Choque \& Mamani, 2015).

En cuanto a adaptación de conducta cerca del 99,1\% de la población de estudio presenta niveles bajo y medio, siendo el clima social familiar una de las influencias en el desarrollo de la adaptación de conducta en los adolescentes.

El clima social familiar posee una alta correlación con la adaptación de conducta en el adolescente. El resultado es el similar que presenta Verdugo et al. (2014) en su investigación titulada "Influencia del clima social familiar en el proceso de adaptación social del adolescente", pues la principal función de la familia es sociabilizar a sus integrantes, es decir, transmitir patrones de conducta. Es por ello que la familia es el sistema donde ocurre el cambio evolutivo de sus integrantes.

El grado de relación que ostentan las variables clima social familiar y adaptación personal, indica relación media y directa; esto se debe a que la familia proporciona al adolescente patrones de comportamiento que lo ayudan a responder ante las exigencias que su ambiente demanda. Un adolescente se siente a gusto consigo mismo y con sus sentimientos, porque la familia incuba los patrones de conducta en la niñez que se formarán en la adolescencia.

\section{Conclusiones}

El 49.1\% de los estudiantes encuestados presenta un nivel promedio con relación al clima social familiar, mientras que el $14.5 \%$, un nivel malo. Asimismo, el $53.6 \%$ de los estudiantes muestra dificultades en su adaptación de conducta y tan solo el 9\% muestra una satisfactoria adaptación. Otro resultado muestra el nivel de correlación entre las variables de estudio: clima social familiar y adaptación de conducta $(r=, 514$ $\mathrm{p}<0.05)$, clima social y adaptación personal $(\mathrm{r}=, 347$ $\mathrm{p}<0,05)$, clima social familiar y adaptación familiar $(\mathrm{r}=520 \mathrm{p}<0.05)$, clima social familiar y adaptación social $(r=, 288 p<0.05)$. Sin embargo, se encontró que no existe correlación entre el clima social familiar y la adaptación educativa $(r=-, 005 \mathrm{p}>0,05)$, lo cual indica que el niño adquiere patrones de socialización en la familia que terminan de desarrollarse en otros grupos pares en la adolescencia.

\section{Referencias}

Choque, G. \& Mamani, F. (2015). Juliaca, ciudad abierta. Un eje articulador sureño. 176-194. Obtenido de http:// www.descosur.org.pe/wp-content/uploads/2014/12/ Juliaca_PeruHoy_Dic2012.pdf

Daly, G. (14 de junio de 2015). Los problemas en los centros de reinserción juvenil (INFROME). El Comercio. Obtenido de http://elcomercio.pe/lima/ciudad/ problemas-centros-reinsercion-juvenil-informe-noticia-1818559

Díaz, M. (2013). Adaptación de la conducta en mujeres sometidas a histerectomía (estudio realizado con mujeres sometidas a histerectomía en el Hospital Nacional de Occidente San Juan de Dios de Quetzaltenango). Quetzaltenango: Universidad Rafael Landívar. Obtenido de http://biblio3.url.edu.gt/Tesario/2013/05/42/ Diaz-Maria.pdf

Ethel, A. \& Godiel, V. (2012). Ambiente social familiar asociado a embarazo en adolescentes que acuden al Instituto Nacional Materno Perinatal. Universidad Nacional Jorge Basadre Grohmann, 5-11.

García, C. (2005). Habilidades sociales, clima social familiar y rendimiento académico en estudiantes universitarios. Liberabit. Revista de Psicología, 63-74.

Gutiérrez, F. (2005). Teorías del desarrollo cognitivo. Aravaca: McGraw-Hill.

INEI. (2015). Estado de la Población Peruana 2015. Lima. Obtenido de https://www.inei.gob.pe/media/MenuRecursivo/publicaciones_digitales/Est/Lib1251/Libro.pdf 
Moss, R., Moss, B. \& Trickett, E. (1984). Escalas de clima social familiar. Madrid: Tea Ediciones.

Orantes, L. (2011). Estado de adaptación integral del estudiante de educación media de El Salvador. El Salvador, Universidad Tecnológica de El Salvador. Obtenido de http://www.utec.edu.sv/media/investigaciones/ files/estado_de_adaptacion_integral_del_estudiante_2011.pdf

Pichardo, M., Fernández, E. \& Amezcua, J. (2002). Importancia del clima social familiar en la adaptación personal y social de los adolescentes. Revista de Psicología, 575-589.

Real Academia Española. (2015). Diccionario de la Real Academia Española. Madrid. Obtenido de http://dle. rae.es $/$ ?id $=0$ nrQ4BH

Roth, E. (2000). Psicología ambiental: interfase entre conducta y naturaleza. Revista número 8, 63-67.

Sarason, I. \& Sarason, B. (2006). Psicopatología; psicología anormal: el problema de la conducta inadaptada (undécima edición). México: Pearson Educación de México SA de C.V. Obtenido de https://books.google.com. pe/books?id=U9aZ9cSXuRoC\&pg=PA $8 \&$ dq $=$ la + - adaptaci $\% \mathrm{C} 3 \% \mathrm{~B} 3 \mathrm{n}+\mathrm{de}+$ conducta $\& \mathrm{hl}=\mathrm{es} \& \mathrm{sa}=\mathrm{X} \&$ ved $=0$ ahUKEwjFnIDD 1 PjKAhVDQSYKHcZyAPEQ6AEIGzAA\#v=onepage \& q = la $\% 20$ adaptaci $\% \mathrm{C} 3 \% \mathrm{~B} 3 \mathrm{n} \% 20 \mathrm{de} \% 20$ conducta $\& \mathrm{f}=$ false

Sausa, M. (6 de septiembre de 2014). La depresión causa el $80 \%$ de suicidios en el Perú, pero no es atendida. Perú 21. Obtenido de http://peru21.pe/actualidad/depresion-ciberbullying-sis-instituto-nacional-salud-mental-honorio-delgado-hideyo-noguchi-2197688

Vargas, J. (2009). Percepción del clima social familiar y actitudes ante situaciones de agravio en la adolescencia tardía. Interdisciplinaria, 289-316.

Vera, J., Morales, D. \& Vera, C. (2005). Relación del desarrollo cognitivo con el clima familiar y el estrés de la crianza. Psico-USF, 161-168.

Verdugo, J., Arguelles, J., Guzmán, J., Márquez, C., Montes, R. \& Uribe, I. (2011). Influencia del clima familiar en el proceso de adaptación social del adolescente. Psicología desde el Caribe, 2018-222.

White, E. (1984). Mensaje para los jóvenes. Florida: Asociación Casa Editora Sudamericana. 